During the impact of the $\mathrm{W}$ fragment, the Galileo spacecraft and HST were observing simultaneously through nearly identical filters; both detected a signal near the limb of the planet. The bright, rapid flash seen by Galileo's cameras was interpreted as the incoming bolide, but from HST's vantage point, this should have been behind the planet's limb, and hence invisible. What did the HST detect? One possibility is a flash reflected off either a dust trail following behind the comet fragment, or a cloud of high altitude debris deposited by dust which preceded the fragment into Jupiter's atmosphere. Alternatively, HST may have detected the beginning of the fragment's glowing, meteor-like interaction with Jupiter's thin upper atmosphere.

Atmospheric waves were detected immediately following the impacts of A, $\mathrm{E}, \mathrm{G}, \mathrm{Q1}$, and R; the HST images revealed concentric rings spreading out from the largest impact sites, yielding a wave-front speed of $454+/-20 \mathrm{~m} / \mathrm{sec}$. The rings spread more slowly than the slowest acoustic wave, which propagates at the altitude of the temperature minimum at $800 \mathrm{~m} / \mathrm{s}$. Gravity waves bracket the observations, but the mechanism that selects out the particular speeds that were observed has not been identified. By successfully matching the observations, we may learn more about the vertical distributions of ambient temperature and energy release from the impacts.

The ballistic ejecta forming the plumes fell back on the upper stratosphere of Jupiter, heating huge areas of atmosphere, typically about the diameter of the Earth, or more. As these huge hot clouds of debris rotated into view from the Earth, they produced immense infrared brightenings and large dark clouds seen by ground-based observers. The morphology of the debris clouds should provide clues to the impactors' penetration depths, and thus, their sizes. We discuss both the evolution of selected sites by the jovian winds, and the overall change in Jupiter's appearance as a result of the bombardment.

\title{
HUBBLE SPACE TELESCOPE FAR-ULTRAVIOLET IMAGING OF JUPITER DURING THE IMPACTS OF COMET SHOEMAKER-LEVY 9
}

J.T. Clarke (U. Michigan, Ann Arbor), R. Prangé (IAS, Orsay), G.E. Ballester (U. Michigan, Ann Arbor), J. Trauger (JPL), D. Rego (IAS, Orsay), R. Evans, K. Stapelfeldt (JPL), W. Ip (MPI Aeronomie, Katlenburg-Lindau), F. Paresce (STSCI, Baltimore), J.C. Gérard (Inst. Astrophys. Liege), H. Hammel (MIT), M. Ballav (U. Michigan, Ann Arbor), L. Ben Jaffel (IAP, Paris), J.L. Bertaux (S.A., Verriéres), D. Crisp (JPL), C. Emerich (IAS, Orsay), W. Harris (U. Michigan, Ann Arbor), M. Horanyi (LASP, Boulder), 
S. Miller (U. College, London), A. Storrs (STScI, Baltimore), H. Weaver (STScI, Baltimore)

We present Hubble Space Telescope (HST) far-ultraviolet images of Jupiter over 13 July - 9 August 1994 during the period of impacts of Comet ShoemakerLevy 9 with the Wide Field Planetary Camera (WFPC2) and the Faint Object Camera (FOC). We have imaged Jupiter at far-ultraviolet (FUV) wavelengths less than 2100 Angstroms to study the response of Jupiter's upper atmosphere to the impacts, to measure upper atmospheric winds through observed motions of impact-related absorbers, and to search for auroral emissions associated with the cometary material passing through Jupiter's magnetosphere or from the impacts. We have detected extraordinary extended absorption regions at all large impact sites in the Rayleigh scattered solar continuum over 1700-2100 A. In the FUV images the impact regions were considerably darker, more extended, and more diffuse with less central contrast than at longer wavelengths, and were observed to actively darken over 2-3 hours post-impact. This indicates the presence of gases or aerosols which were more spatially extended, had a stronger absorption in the FUV than at visible wavelengths, and were likely to be at higher altitudes. Although the impact feature centers remained generally fixed in system III longitude, the initial dispersions of FUV absorbing material in the $G, L$, and $K$ features showed stronger absorptions and more pronounced north/south extensions, with initial motions of the order of $1 \mathrm{~km} / \mathrm{sec}$. We have detected transient northern auroral emissions at unusually low magnetic latitudes just after the $K$ impact, with similar but fainter emissions at either side of the $\mathrm{K}$ impact site in the southern hemisphere. The northern emissions appeared to fall near the magnetic conjugate point of the $\mathrm{K}$ impact site along $2 \mathrm{RJ}$ magnetic field lines, initially with $40 \mathrm{kR}$ brightness and decreasing with time. We have noted fainter than average global auroral activity before and during the period of collisions, which returned to a normally bright level several days after the end of the impacts, although its relationship to the event is not straightforward. Finally, we present a variable auroral emission feature along the 'normal' southern auroral oval roughly $1 / 2$ hour before the P2 impact.

\section{HST SPECTROSCOPIC OBSERVATIONS OF JUPITER AFTER THE COLLISION OF COMET P/SHOEMAKER-LEVY 9}

K.S. Noll, M.A. McGrath (STScI, Baltimore), L.M. Trafton (U. Texas, Austin), S.K. Atreya (U. Michigan, Ann Arbor), J.J. Caldwell (Space Astrophys. Lab., North York, Ontario), H.A. Weaver (STScI, Baltimore), R.V. 